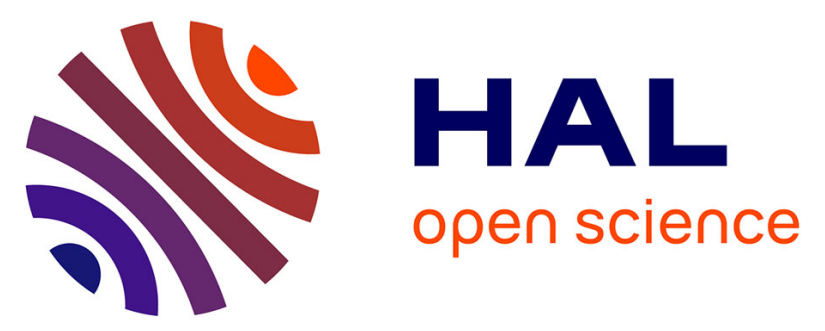

\title{
Nanoimprinted, Submicrometric, MOF-Based 2D Photonic Structures: Toward Easy Selective Vapors Sensing by a Smartphone Camera
}

Olivier Dalstein, Davide R. Ceratti, Cédric Boissière, David Grosso, Andrea Cattoni, Marco Faustini

\section{To cite this version:}

Olivier Dalstein, Davide R. Ceratti, Cédric Boissière, David Grosso, Andrea Cattoni, et al.. Nanoimprinted, Submicrometric, MOF-Based 2D Photonic Structures: Toward Easy Selective Vapors Sensing by a Smartphone Camera . Advanced Functional Materials, 2016, 26 (1), pp.80. 10.1002/adfm.201670006 . hal-01253850

\section{HAL Id: hal-01253850 https://hal.sorbonne-universite.fr/hal-01253850}

Submitted on 11 Jan 2016

HAL is a multi-disciplinary open access archive for the deposit and dissemination of scientific research documents, whether they are published or not. The documents may come from teaching and research institutions in France or abroad, or from public or private research centers.
L'archive ouverte pluridisciplinaire HAL, est destinée au dépôt et à la diffusion de documents scientifiques de niveau recherche, publiés ou non, émanant des établissements d'enseignement et de recherche français ou étrangers, des laboratoires publics ou privés. 
DOI: 10.1002/ ((please add manuscript number))

Article type: Full Paper

\title{
Nano-imprinted, Sub-micrometric, MOF-based 2D Photonic Structures: Toward Easy Selective Vapors Sensing by a Smart-Phone Camera
}

Olivier Dalstein, Davide R. Ceratti, Cédric Boissière, David Grosso, Andrea Cattoni, * Marco Faustini, *

O. Dalstein, D.R. Ceratti, Dr. C. Boissiere, Pr. D. Grosso, Dr. M. Faustini Sorbonne Universités, UPMC Univ Paris 06, CNRS, Collège de France, UMR 7574, Chimie de la Matière Condensée de Paris, F-75005, Paris, France

E-mail:marco.faustini@upmc.fr

Dr. A. Cattoni, Pr. D. Grosso

Laboratoire de Photonique et de Nanostructures, LPN, Route de Nozay, 91460 Marcoussis, France

E-mail:andrea.cattoni@1pn.cnrs.fr

Pr. D. Grosso

M2NP, Faculté des Sciences et Techniques, Campus de Saint Jérôme, Avenue Escadrille Normandie Niemen, 13397 Marseille, France

\begin{abstract}
In this work we describe a soft-lithographic approach to fabricate sub-micrometer MOFbased 2D photonic structures. Nanometric Zeolitic Imidazole Framework material ZIF-8 (zinc) was chosen as the sensible MOF material because of its chemical stability and its vapor selective adsorption properties. Two different systems were fabricated: nanopatterned colloidal ZIF-8 homo- and $\mathrm{ZIF}-8 / \mathrm{TiO}_{2}$ hetero-structures. Several features (stripes, squares, etc.) with dimension as small as $200 \mathrm{~nm}$, were replicated on different substrates such as silicon, flexible plastics and even on aluminum cans, over relatively large surfaces (up to 1 $\mathrm{cm}^{2}$ ). In addition, we demonstrate here that these photonic MOF-heterostructures can be used as very low-cost sensing platforms compatible with smart-phone technology. This method relies on the evaluation of the change in diffraction efficiency of the photonic MOF-patterns, induced by the MOF refractive index variation, which is simply detected by a CCD camera,
\end{abstract}


as those integrated in simple smart-phones, without need of complex optical instrumentations for transduction data processing. Performances of the sensors were first evaluated using isopropanol adsorption/desorption cycling as a model case. In addition, a "real" environmental issue was tackled. Selective detection of styrene in presence of interfering water was demonstrated at concentration below the human permissible exposure limit. In-situ ellispometric analyses were also carried out in order to confirm the sensor performances and to propose a mechanism for styrene uptake into the nanoMOFs.

\section{Introduction}

Metal Organic Frameworks (MOFs) have attracted immense attention because of their large, tunable porosity and versatile chemistry ${ }^{[1]}$ that makes them appealing for many applications, including storage, separation, ${ }^{[2]}$ sensing $^{[3]}$ and catalysis. ${ }^{[4]}$ However these materials are generally synthetized under powder form that is not always suitable for direct application. In order to fulfill their promises, MOFs need to be processed, shaped and integrated in functional devices. ${ }^{[5]}$ This is especially the case for the utilization of MOFs in the domain of photonics. Some recent works demonstrated the integration of MOFs into photonic components such as thin films, ${ }^{[6]}$ Fabry-Pérot, ${ }^{[7]}$ 1D Bragg stacks, ${ }^{[8]}$ or $3 \mathrm{D}$ colloidal crystals. ${ }^{[9]}$ In all these examples the materials were exploited as chemical sensors, ${ }^{[10]}$ for which the signal transduction relies on the change of refractive index in response to the analyte adsorption into the porosity. ${ }^{[6]}$ On the other hand, the integration of MOFs into 2D photonic components haven't been explored yet. Two dimensional periodical systems are common architectures of many, sophisticated nanophotonic devices such as 2D photonic crystals, gratings, waveguides, plasmonic antenna ${ }^{[11]}$ or even metamerials. ${ }^{[12]}$ A simple method for the integration of MOFs into $2 \mathrm{D}$ photonic components would pave the way for advanced optical devices in which the original properties of MOFs (high porosity, sorption selectivity..) and of the periodic structures (light confinement, diffraction, guiding..) are rationally combined. The main technological challenge to further develop such devices is that they require fabrication of nonscattering, patterned films at the sub-micrometer scale. With such a perspective, the seemingly simple step aiming at patterning MOFs materials by lithographic approaches has become a very active research domain in the last years and several strategies have been proposed. $^{[13]}$ MOF crystals have been 2D spatially localized by soft-lithographic approaches, ${ }^{[14]}$ digital microfluidics, ${ }^{[15]}$ localized electrochemical, ${ }^{[16]}$ growth, ${ }^{[17]}$ seedmediated $^{[18]}$ deposition, Dip-Pen Lithography, ${ }^{[19]}$ Deep X-Ray Lithography, ${ }^{[20]}$ UV 
Lithography ${ }^{[21]}$ combined with hot embossing. ${ }^{[22]}$ However, none of these approaches was suitable for fabricating MOFs-based 2D photonic devices since crystals size and/or pattern periodicity were in the micrometer range. In addition, most of the reported patterning methods were not adapted to large-scale fabrication on different substrates, a critical aspect to enable their utilization as sensing platform for instance.

Here we propose a simple and versatile approach to fabricate sub-micrometer MOF- based 2D photonic structures, and we demonstrate their utilization as very low-cost sensing platforms compatible with smart-phone technology. Soft Nano-Imprinting Lithography was exploited as patterning technology because of its easiness, versatility and because it allows fabrication at throughputs necessary for many realistic applications. Zeolitic Imidazole Framework material ZIF-8 (zinc) was chosen as the sensible MOF material. ${ }^{[23]}$ In general, ZIFs are chemically and thermally stable materials, a fundamental requirement for any practical device. Study of the gas adsorption and thermal and chemical stability of ZIF-8 ( $\mathrm{Zn}$ ) demonstrated its permanent, high thermal stability (up to $550^{\circ} \mathrm{C}$ ), and remarkable chemical resistance to boiling alkaline water and organic solvents. ${ }^{[24]}$ In addition, due to its great surface area and "large porosity", ZIF-8 (Zn) is the most used in chemical sensing because of the excellent selective adsorption properties. ${ }^{[23]}$ In order to obtain optical quality patterns and overcome light-scattering, the material was processed as colloidal solution in which the crystal sizes were inferior to $50 \mathrm{~nm}$. Two different systems were fabricated: nanopatterned colloidal ZIF-8 homo- and $\mathrm{ZIF}-8 / \mathrm{TiO}_{2}$ hetero-structures. Both patterned systems were obtained by either nanoimprinting directly a sessile drop of nano-MOF suspension, or by depositing this suspension onto a nanoimprinted sol-gel based titania films. Several features (stripes, squares, etc.) with dimension as small as $200 \mathrm{~nm}$, were replicated on different substrates such as silicon, flexible plastics and even on aluminum cans, over relatively large surfaces (up to 1 $\mathrm{cm}^{2}$ ). The resulting systems exhibited angle-dependent structural colors characteristic of 2D diffraction gratings. A new sensing approach based on the variation of optical properties of MOF patterns, induced by the selective adsorption of organic solvent vapors, was investigated. This method relies on the evaluation of the adsorption-induced-color-luminance change, induced by the MOF refractive index variation, which is simply detected by a CCD camera, as those integrated in simple smart-phones, without need of complex optical instrumentations for transduction data processing. Proof of concept and performances were first demonstrated using isopropanol adsorption/desorption cycling as model system. It was then applied to the real industrial problem of the production of polystyrene latex prepared by oil in water emulsions of styrene, for which the detection of reactor leaks and quantitative 
analysis of a highly toxic styrene in presence of high concentration of interfering water is a "real" safety issue. We demonstrated that styrene could be selectively detected and quantified by evaluating its adsorption kinetic by the patterned MOF layer. Concentrations as small as $57 \mathrm{ppm}$, which is below the human permissible exposure limit, could be detected. In all cases, the styrene adsorption was evaluated by optical transduction using the associated luminance variation of the gratings probed by a simple CCD camera, and double checked by direct analysis of the optical properties of a non-patterned film through conventional spectroscopic ellipsometry.

\section{Results and discussion}

\subsection{Fabrication of the patterns}

The nanopatterning strategy of porous ZIF-8 materials is based on the utilization of preformed ZIF-8 nanocrystals as illustrated in Figure 1. ZIF-8 nanoparticles suspension was prepared by following the procedure previously reported by Cravillon et al. ${ }^{[25]}$ The reaction leads to a stable and homogeneous colloidal solution of microporous ZIF-8 nanocrystals in ethanol. The crystal average size was measured equal to $41 \pm 8 \mathrm{~nm}$ by scanning electron microscopy (Fig. S1). Thin films exhibiting high optical quality could be obtained by dip coating or spin coating the colloidal solution on silicon substrates. The crystal structure of the particles films was accessed by Grazing-Incident Wide-Angle X-ray Scattering (GI-WAXS) that shows the characteristic pattern of ZIF-8 materials (Fig. S2). The refractive index of the colloidal films was measured to be equal to $1.2 \pm 0.05$ depending on particle's packing, in agreement with previous findings. ${ }^{[6]}$ In order to characterize the porosity and the vapor sorption selectivity of the colloidal ZIF-8 films, water and isopropanol adsorption experiments have been carried out at room temperature (Fig. S3). The adsorption experiments were performed by environmental ellipsometric porosimetry (EEP) on a non-patterned film having a thickness of $155 \mathrm{~nm}$ obtained by dip-coating the ZIF colloidal solution at $5 \mathrm{~mm} \cdot \mathrm{s}^{-1}$ onto a silicon substrate. The hydrophobicity of the ZIF-8 microporosity was confirmed since no significant variation in refractive index is observed except for very high vapor pressure $\left(\mathrm{P} / \mathrm{P}_{0}>0.9\right)$, for which water condensation occurs in the inter-particle voids. On the other hand, isopropanol (i-PrOH) adsorption curve shows a double steps increase of refractive index at low and high relative pressures, due to $\mathrm{i}-\mathrm{PrOH}$ adsorption into the micropores of the ZIF-8 framework (17\% of the total film volume) and the inter-particular mesopores $(39 \%$ of the 
total film volume) as observed in a previous study. ${ }^{[6 a]}$ The quantitative analysis of the adsorbed volume fraction was obtained through the BEMA (Bruggeman Effective Medium Approximation) model assuming the refractive index of the dense ZIF-8 material being equal to $1.54 .^{[6 \mathrm{a}]}$ In addition, the ellipsometric investigation indicated that capillary condensation into the ZIF-8 micropores is associated with a weak contraction of the film (around 2\%, data not shown).

Concerning nanostructuration of the ZIF-8 nano crystals films, two routes were selected. In the first one, soft nanoimprint lithography was applied directly on a drop-casted ZIF-8 colloidal suspension (see Figure 1a). In the second one, ZIF-8 colloids are deposited onto a pre-patterned $\mathrm{TiO}_{2}$ layer (see Figure 1b).

(a)
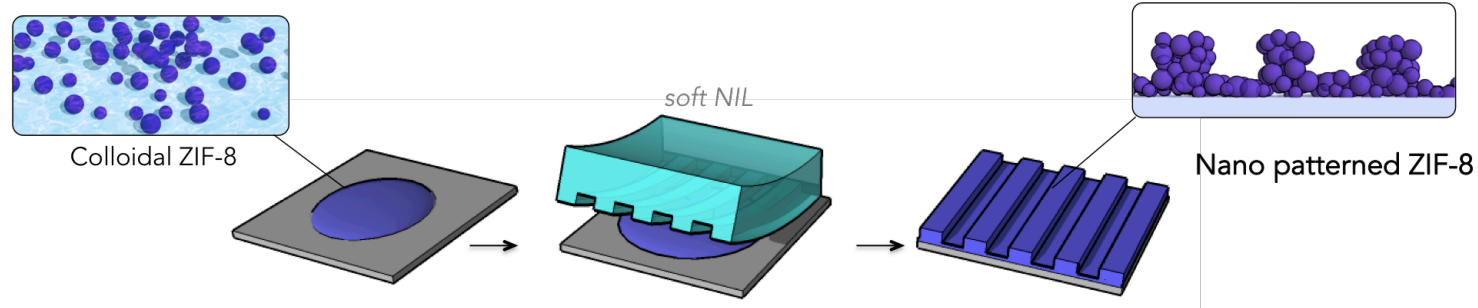

(b)
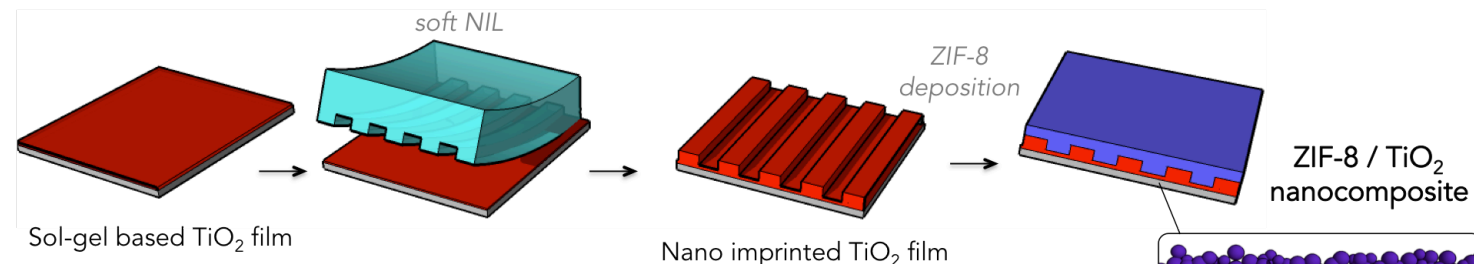

Nano imprinted $\mathrm{TiO}_{2}$ film

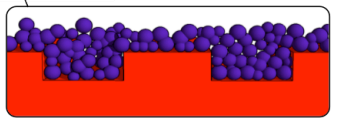

Figure 1 Soft-lithographic approaches for the fabrication of (a) nanopatterned ZIF-8 and (8) ZIF-8/ $/ \mathrm{TiO}_{2}$ heterostructures.

For the first route, a $10 \mu \mathrm{L}$-droplet of colloidal solution was first applied on the substrate. The solution droplet was immediately covered by the patterned PDMS stamp without applying any external pressure. The liquid layer was homogeneously spread beneath the PDMS stamp while the stamp's nanocavities were naturally filled by capillarity. In this configuration, the evaporation of the solvent takes place through the permeable PDMS stamp. Replication of the features and homogeneous packing of the colloidal network were obtained after complete evaporation and de-molding. Finally, the system was consolidated at $130^{\circ} \mathrm{C}$ for 10 minutes. 
An example of replication is shown on the optical photograph in Figure 2(a) that displays a $1 \mathrm{~cm}^{2}$ pattern on silicon substrate exhibiting bluish diffracted color due to the presence of a grooves array shown in the SEM micrograph of Figure 2(d). The imprinted patterns are composed of randomly packed ZIF-8 nanocrystals, as shown in the higher magnification SEM photos in Figure 2 (f) and Figure 2(g) (and schematically represented in Figure 1 (a)). The periodicity of the grooves array was equal to $780 \mathrm{~nm}$ while both width and height of the lines ranged between 200 and $240 \mathrm{~nm}$ (corresponding to 5-6 colloids packed together). A 200nmthick residual layer composed by a continuous film of ZIF-8 colloids remained at the bottom. The thickness of the residual layer could be partially tuned by modifying the concentration of the initial ZIF-8 colloidal solution. Figure 2(h) shows the cross-section SEM micrograph of a sample obtained from the same replication process but by diluting twice the initial colloidal solution. In this configuration, grooves array that are free of residual layer are obtained. Other pattern morphologies such as square arrays, as displayed in SEM micrograph in Figure 2(c), could be obtained using appropriated PDMS stamps. Being based on the replication of flexible stamp, the method could be extended on a wide range of substrates such as a curved aluminum can (Fig. 2b) or a transparent, flexible polypropylene film (Fig 2c). In these cases the morphology and the quality was similar to those observed on silicon in Figure 2(d).
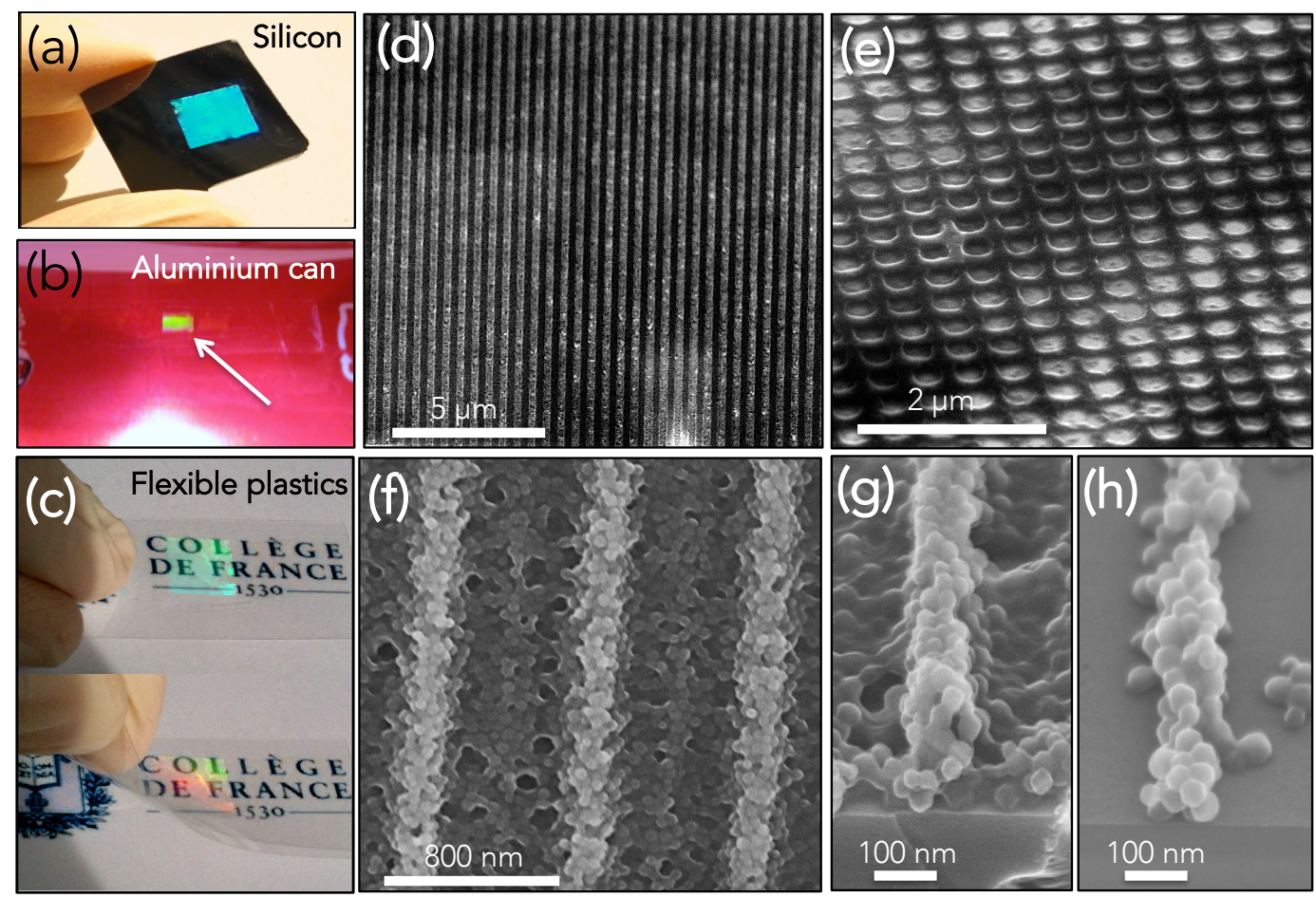

Figure 2 Photograph of the diffracting ZIF-8 nanopatterned surfaces applied on (a) a silicon wafer, (b) an aluminum can and (c) a flexible polypropylene film. FEG-SEM micrographs of 
patterned ZIF-8 colloids showing square (e) and nanogrooves (d) features of at different magnifications (f), (g) and without residual layer (h).

Figure 1(b) displays the second fabrication route for the preparation of nanostructured $\mathrm{TiO}_{2} / \mathrm{ZIF}-8$ multilayer coatings. The fist step consisted in the deposition of sol-gel based titania layer by spin-coating on silicon. Immediately after evaporation of the volatile solvent, the uncondensed titania precursors layer was embossed by a flexible hard-PDMS/PDMS stamp without applying any pressure using a technique called Degassing Assisted Patterning applied to the direct embossing of sol-gels derived films. ${ }^{[26]}$ The system was stabilized at $110^{\circ} \mathrm{C}$ for 5 minutes before demolding, and before being heat-treated at $450^{\circ} \mathrm{C}$ for 5 minutes to ensure complete densification and crystallization of $\mathrm{TiO}_{2}$ network. The nanopatterned $\mathrm{TiO}_{2}$ film is thus composed by a dense network of sub-20 nm anatase particles as characterized by SEM and GI-WAXS and shown in Fig. S4 The cross-section view of a typical nanoimprinted $\mathrm{TiO}_{2}$ film with periodicity of $400 \mathrm{~nm}$ is shown in Fig. 3(a). The nanopatterned $\mathrm{TiO}_{2}$ films were then homogenously covered by ZIF-8 colloids by dip-coating. The morphology of the final nanostructured $\mathrm{TiO}_{2} / \mathrm{ZIF}-8$ system is shown on the SEM photos of Figure 3(b) and (c). In order to better visualize the whole system a band of the ZIF-8 layer was wiped away from the surface (see Figure 3 (a)), and clearly revealed the bottom $\mathrm{TiO}_{2}$ pattern, consisting in an array of lines with periodicity equal to around $1000 \mathrm{~nm}$ in this case. In this configuration, the ZIF-8 layer covered the whole surface as testified by SEM images of Figure 3(c) and Figure S4. The thickness of ZIF-8 colloids layer over the lines was evaluated by SEM being equal to $180 \mathrm{~nm}$ it could be easily tuned by controlling the deposition conditions.
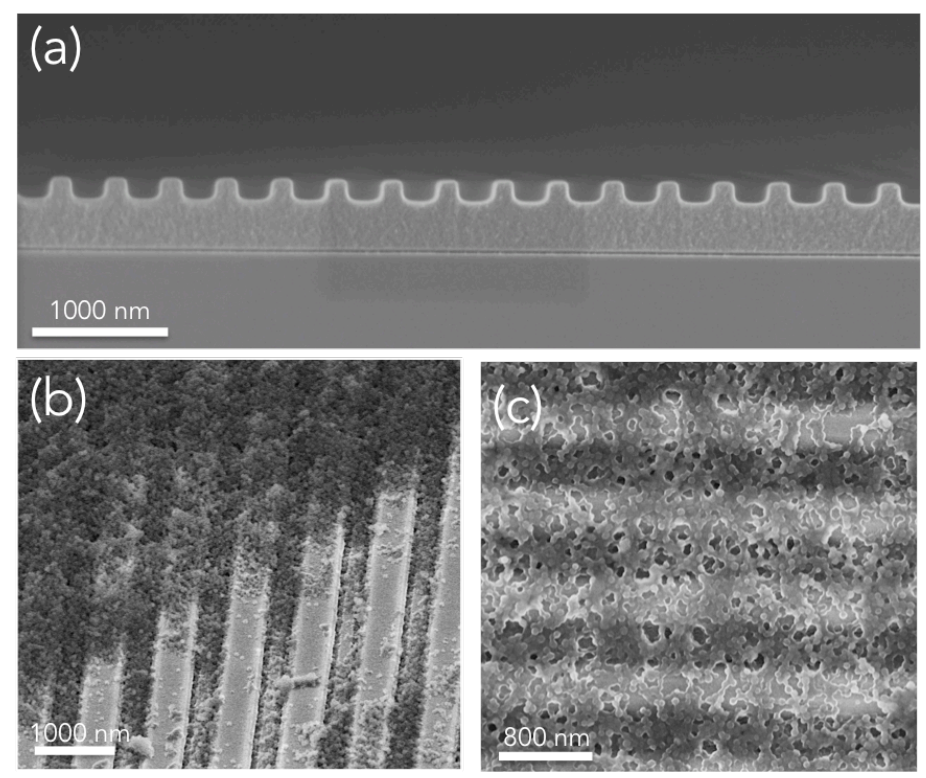
Figure 3 FEG-SEM micrographs of (a) typical patterned $\mathrm{TiO}_{2}$ film (cross-section view) and ZIF-8 $/ \mathrm{TiO}_{2}$ hetero-structures: (b) scratched zone exhibiting both ZIF-8 covered and uncovered $\mathrm{TiO}_{2}$ nanogrooves and (c) higher magnification of the deposited zone.

\subsection{MOF-based diffraction grating sensors}

The MOF patterned surfaces have been used as reflective diffraction gratings enabling easy sensing by a simple smart-phone's camera. The principle is illustrated in Figure 4(a). When the periodical structure is irradiated by a white light, a part of the reflected light is split by diffraction into several beams travelling in different directions. The directions of the beams depend on the spacing of the grating and the wavelength. When light is normally incident on the gratings, the relationship between the grating spacing $d$ and the angle of the diffracted beams of light $\theta_{m}$ is given by the following Bragg's equation (1):

$d \sin \theta_{m}=m \lambda$

where $m$ is the diffraction order and $\lambda$ the wavelength of each component of the light. The top image in Figure 4(b) shows the photograph of the ZIF-8 gratings having d-spacing of 1000 $\mathrm{nm}$ taken by the photo-camera of commercially available smart-phone. The image was taken at angle $\theta_{m}$ equal to $28^{\circ}$ as respect to incident beam. In this configuration, the color of the pattern looks blue since the $1^{\text {st }}$ order diffracted light has a wavelength equal to $469 \pm 5 \mathrm{~nm}$ according to equation 1 . The photo taken by the camera is then converted into gray-scale format. The process consists in the conversion of the RGB values to Luminance Intensity for each pixel. The image analysis of a small Zone Of Interest (ZOI) gives an average value of Luminance Intensity that can be directly attributed to the efficiency of the diffracting platform. If one assumes that the height of the nanostructures does not vary, the intensity of the diffracted light depends on the refractive index contrast between the patterned material and the surrounding medium. Opposite tendencies are thus expected for the two ZIF- 8 based systems illustrated in Figure 4 (c) while exposed to organic vapors. In the case of directly patterned ZIF-8 colloids, the MOF layer plays the role of "active" diffraction grating. One expects an increase in diffraction luminance created by the increase of the optical index contrast between the medium $\left(\mathrm{n}_{\mathrm{air}}=1\right.$ : constant) and the grating material $\left(\mathrm{n}_{\mathrm{MOF}}=1.2\right.$ : increasing with the organic vapor adsorption). In this case, the modification of the structure 
height is negligible, since we detected a contraction of only $2 \%$ in volume of the MOF layer by ellipsometry at high vapor pressure. In the case of $\mathrm{ZIF}-8 / \mathrm{TiO}_{2}$ heterostructure, the dense $\mathrm{TiO}_{2}$ bottom layer plays the role of "passive" diffraction grating. The uptake of organic vapors by the ZIF-8 porous crystals provokes thus a decrease in optical index contrast between the two components and a decrease in luminance intensity.

(a)

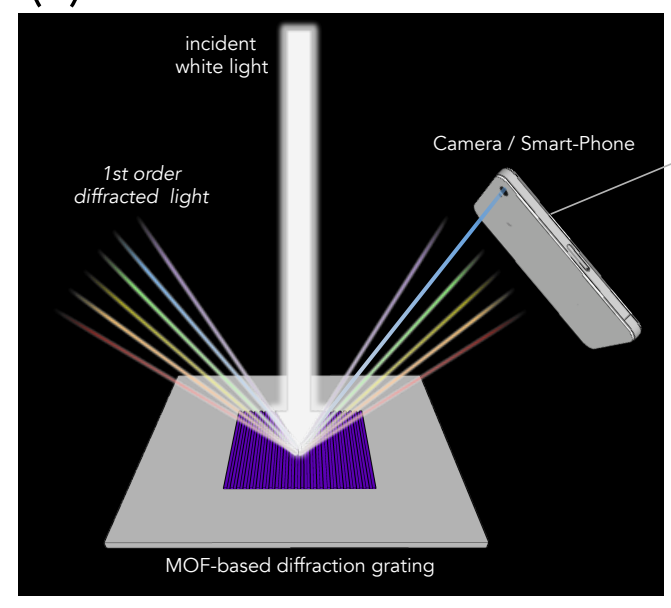

(b)

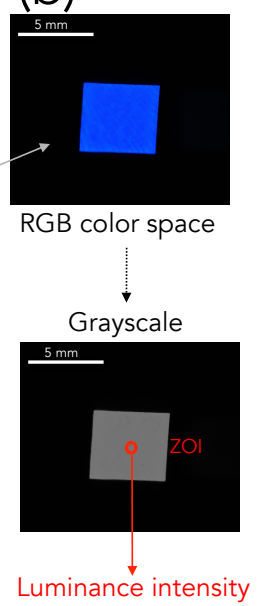

(c)
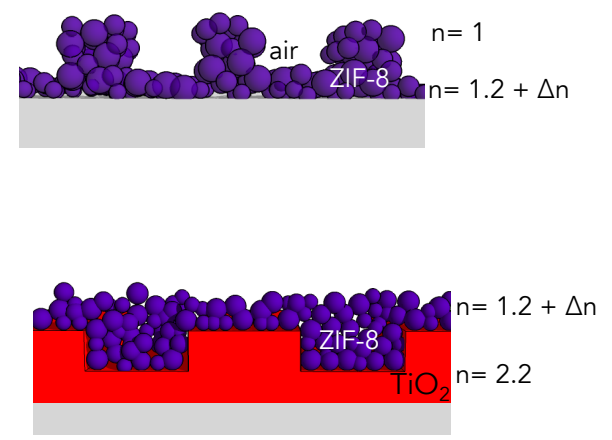

Figure 4 (a) Illustration of the experimental set-up for the detection by a simple camera; (b) photographs of the diffracting grating in colors and gray-scale taken a smart phone photocamera; illustration of the patterned $\mathrm{ZIF}-8$ and $\mathrm{ZIF}-8 / \mathrm{TiO}_{2}$ heterostructure with opposite refractive index evolution as function of the vapor adsorption.

The optical responses of the diffraction grating systems have been first tested using i-PrOH vapor as adsorbate. Both nanopatterned $\mathrm{ZIF}-8$ and $\mathrm{ZIF}-8 / \mathrm{TiO}_{2}$ gratings were composed of grooves, having periodicity of $1000 \mathrm{~nm}$. A pure nanopatterned $\mathrm{TiO}_{2}$ was taken as reference. The variation of the normalized Luminance Intensity with respect to the i-PrOH vapor pressure is shown in Figure 5(a). The two opposite tendencies expected were confirmed. At moderate $\mathrm{i}-\mathrm{PrOH}$ relative vapor pressures, below $\mathrm{P} / \mathrm{P}_{0}=0.6$, an increase of luminance is observed with pure ZIF-8, against a decrease in the case of $\mathrm{ZIF}-8 / \mathrm{TiO}_{2}$ gratings. No significant variation in luminance is observed for a reference $\mathrm{TiO}_{2}$ grating alone. In the case of nanopatterned ZIF-8 gratings, the first luminance increase (I $\rightarrow$ II) is attributed to the vapor uptake into the microposity while a second and $(\mathrm{III} \rightarrow \mathrm{IV})$ is due to the i-PrOH condensation in the interparticles voids. For high i-PrOH vapor pressures, a significant decrease in luminance is observed due to the irreversible collapse of the nanostructure as illustrated in Figure 5 (b) and shown in the SEM micrograph in Fig. S9. No desorption behavior was thus recorded due to the loss of the diffraction efficiency of the sample. In the case of $\mathrm{ZIF}-8 / \mathrm{TiO}_{2}$ 
heterostructure, three decreases in luminance are observed: a first one at low $\mathrm{P} / \mathrm{P}_{0}$ that is attributed to the vapor uptake into the ZIF-8 microporosity (transition I $\rightarrow$ II); an additional decrease below $\mathrm{P} / \mathrm{P}_{0}=0.7$ (transition $\mathrm{II} \rightarrow \mathrm{III}$ ) probably due to the $\mathrm{i}-\mathrm{PrOH}$ condensation at the ZIF-8/ $\mathrm{TiO}_{2}$ interface; and a significant decrease in luminance at $\mathrm{P} / \mathrm{P}_{0}>0.9$ (transition III $\rightarrow$ IV) due to isopropanol capillary condensation within the ZIF-8 interparticles voids. The desorption branch follows the adsorption one except at low pressures where a hysteresis is depicted. This can be attributed to a slower desorption due to the stabilization of the adsorbate within the MOF microporosity. The great difference in luminance between low and high $\mathrm{P} / \mathrm{P}_{0}$ exposure is highlighted in photographs taken by smart phone in Figure 5 (c) for the ZIF8/ $\mathrm{TiO}_{2}$ sample. One must underline here the better stability of the $\mathrm{ZIF}-8 / \mathrm{TiO}_{2}$ heterostructure resulting from the encapsulation of the MOF particles into the hard $\mathrm{TiO}_{2}$ grooves. Differently from the nanopatterned ZIF-8 system, here the patterned material is $\mathrm{TiO}_{2}$ that is insensitive to the i-PrOH exposure. The interparticle condensation occurs in the top ZIF-8 layer but after drying the system recovers its initial morphology (Fig. S9). For this reason, only the results of the $\mathrm{ZIF}-8 / \mathrm{TiO}_{2}$ gratings will be presented from this point hereafter.

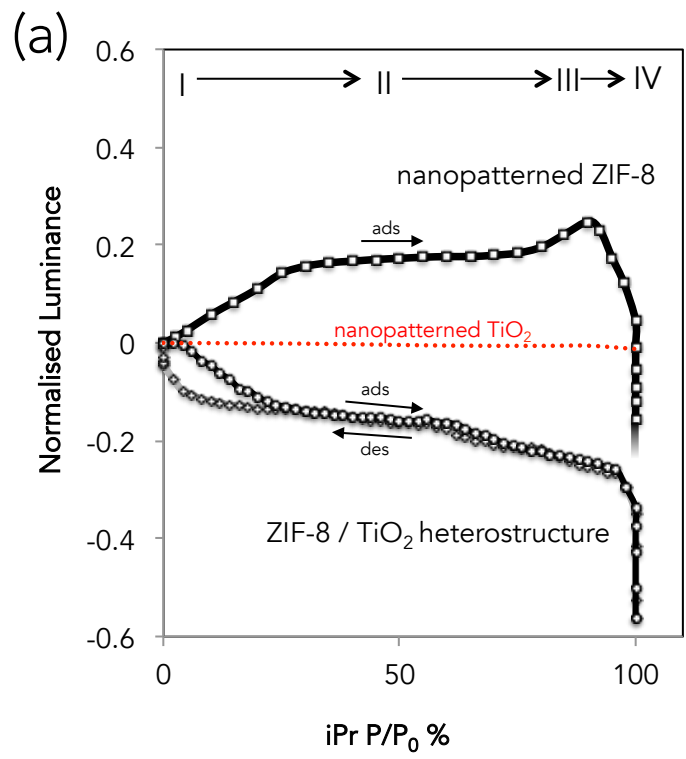

(b)
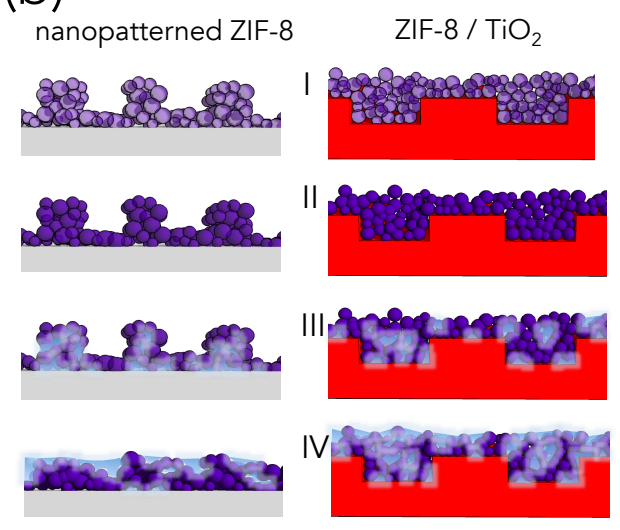

(c)

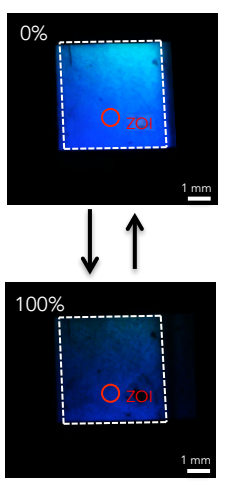

Figure 5 (a) Isopropanol uptake measured by luminance difference; (b) illustration of the phenomena taking place during adsorption ; (c) photographs of the $\mathrm{ZIF}-8 / \mathrm{TiO}_{2}$ heterostructure at 0 and $100 \% \mathrm{P} / \mathrm{P}_{0}$ of isopropanol, taken by a smart phone at an angle of $28^{\circ}$.

In order to probe the reversibility of the $\mathrm{i}-\mathrm{PrOH}$ adsorption/desorption in the $\mathrm{ZIF}-8 / \mathrm{TiO}_{2}$ system, the variation of luminance was investigated during cycling in i-PrOH enriched 
atmosphere between $\mathrm{P} / \mathrm{P}_{0}=0$ and $\mathrm{P} / \mathrm{P}_{0}=0.5$ in air at $25^{\circ} \mathrm{C}$ as shown and detailed in Fig. S5. The variation of luminance upon the adsorption/desorption cycles is reproducible, indicating that the material is able to withstand cyclic exposure to the vapor stream. The same results were obtained by cycling i-PrOH on nanopatterned ZIF-8 gratings (at $\mathrm{P} / \mathrm{P}_{0}$ between 0 and 0.5 , irreversible collapsing does not take place) as shown in Fig. S6.

\subsection{Selective Styrene Detection}

The effectiveness of the detection mode described above for isopropanol vapors is now transposed to a more industrially relevant application such as the selective detection of toxic and volatile styrene monomer in presence of water. Styrene is a building block for synthesis of rubbers, plastics, resins, and polyesters in synthetic industry. In particular, the styrene/water biphasic mixture is the main component of the widely used synthesis of polystyrene beads by oil in water emulsion polymerization. ${ }^{[27]}$ As a potential human carcinogen, styrene vapors may lead to irritation of the skin, eyes and the respiratory system. Similar issues have been discussed in the literature: luminescent Eu-MOFs were used to selectively detect explosive 2,4,6-trinitrophenol (TNP) in water or in vapor phase. ${ }^{[3 a, 28]}$ Several materials have been also exploited to detect styrene (and propanol) by adsorption including conductive polymer composites, ${ }^{[29]}$ hydrogen-bond acidic polymers, ${ }^{[30]}$ organoplatinum coatings, ${ }^{[31]}$ monolayer-protected gold nanoparticles, ${ }^{[32]} \mathrm{ZnO}$ nanowires ${ }^{[33]}$ or Pd- gate metal-oxide-semiconductor. ${ }^{[34]}$ However in all these cases the sensitive materials were applied on different detection platform such as surface acoustic wave (SAW) or resistive sensors. In addition they were not very porous and/or not compatible with a simple optical detection. Due to its high porosity and selectivity, a ZIF-8 material emerges as a strong candidate for ambient optical detection of styrene in presence of water.

The adsorption/desorption behavior of nanoZIF-8 exposed to styrene/water vapors was first investigated on planar films by in-situ ellipsometry as illustrated and detailed in Figure S7. The generation of vapors was obtained by mixing a controlled proportion of dry air, and air enriched with styrene and water vapors in order to control the composition of the atmosphere, indicated in term of relative vapor pressures $(1,2,5,10 \%)$ of styrene and water.

The evolution of the refractive index of a ZIF-8 film exposed to styrene/water vapors generated from liquid mixtures composed of 1, 2, 5, and 10\% styrene is shown in Figure 6(a). 
As expected, the refractive index increases due to the selective styrene uptake into the ZIF-8 particles. As a blank, the same experiment was performed only with water vapors and no variation of the refractive index was observed. Heating the coating $5 \mathrm{~min}$ at $130^{\circ} \mathrm{C}$ in dry air allows to fully desorb the molecules and recover the initial state. The increase in refractive index is relatively slow and the system doesn't reach equilibrium even after 35 minutes of experiment. On the other hand, the styrene uptake rate varies with the vapor pressure, being faster at $10 \%$ and slower at $1 \%$. The styrene uptake variations (expressed as normalized refractive index) were then plotted with respect to the square root of time in Figure 6(b). Each uptake is characterized by two successive linear curves, suggesting that the uptake can be described with two successive phenomena that are both limited by diffusion. An adsorption and diffusion mechanism is proposed and illustrated in Fig. S8. The first faster uptake (regime i) could be attributed to the styrene adsorption on the surface of ZIF- 8 nanoparticles. This regime would be limited by the diffusion of styrene from the atmosphere to the surface of the material and it ends when equilibrium is reached. Being in equilibrium, the concentration of styrene on the surface is directly related to the styrene relative vapor pressure. The second uptake behavior (regime ii) is very probably due to the progressive styrene penetration into the microporosity towards the core of the ZIF-8 particles. Regime ii would be limited by the diffusion of styrene from the surface into the microporosity. 

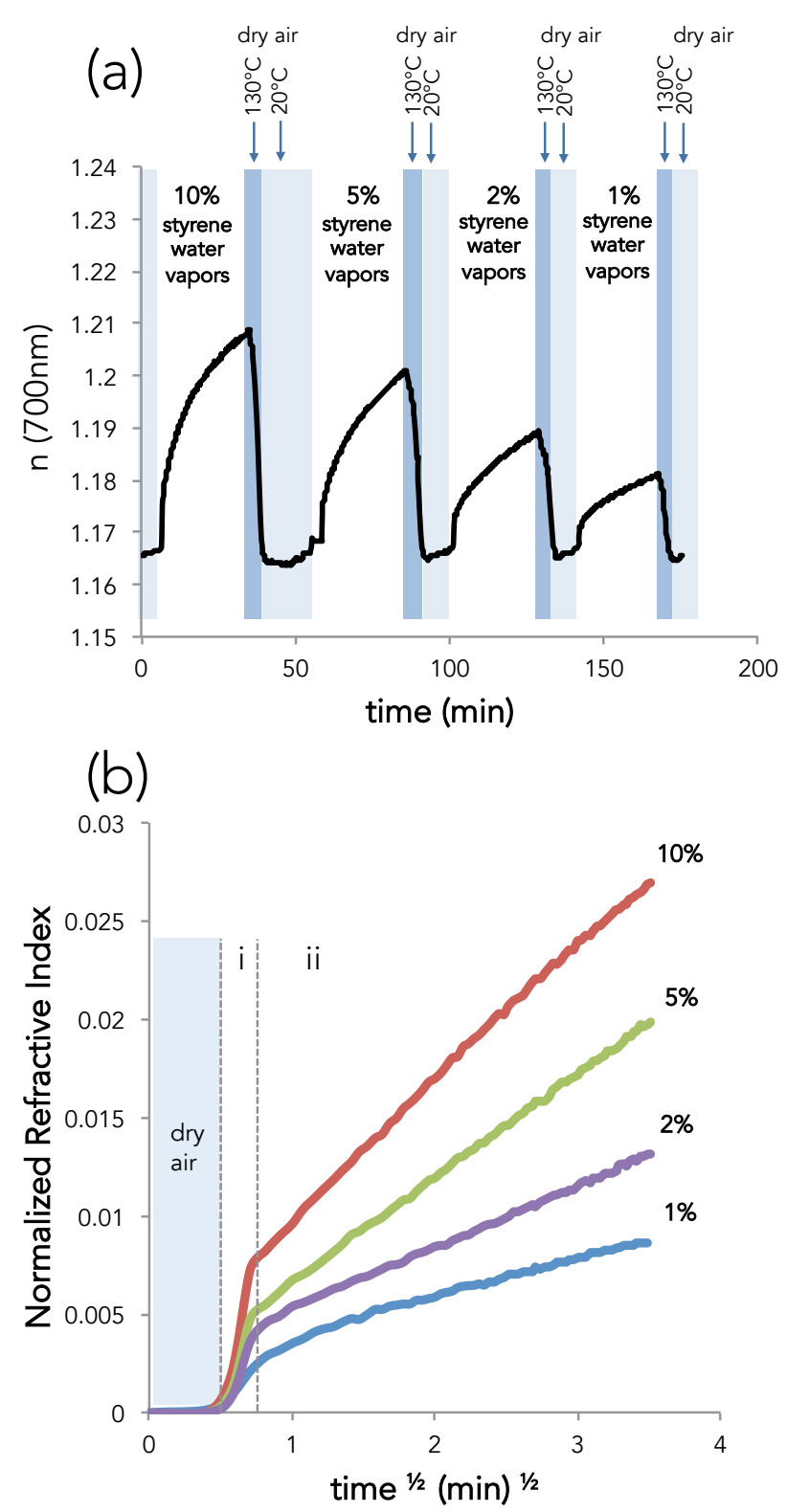

Figure 6 (a) Evolution of the refractive index of ZIF-8 colloidal film with respect to time for styrene/water vapors adsorption/desorption at different concentration. (b) Plot of the normalized refractive index as a function of the square root of the time for styrene/water adsorptions at different vapors concentrations.

Same detection experiments were also carried out by measuring luminance changes using the $\mathrm{ZIF}-8 / \mathrm{TiO}_{2}$ heterostructure as a sensible platform. The evolution of the normalized luminance as function of the square root of the time is shown in Figure 7 (a). As in the case of isopropanol, the luminance decreases while styrene adsorbs in the film. Similarly to what was observed by ellipsometry, two successive linear regimes are identified, probably due to styrene adsorption onto the surface (regime i) and into the microporosity (regime ii). In both 
ellipsometry and luminance measurements, the concentration-dependent uptake rate can be determined from the slopes of the straight tendencies of regime ii. Figure 7(b) shows the plot of uptake rates as a function of the vapors composition obtained from ellipsometry and diffraction grating (DG) experiments. A good agreement in term of tendency is found indicating that sensing of styrene can be performed by using an extremely sensitive technique like ellipsometry as well as a simple photo-camera.
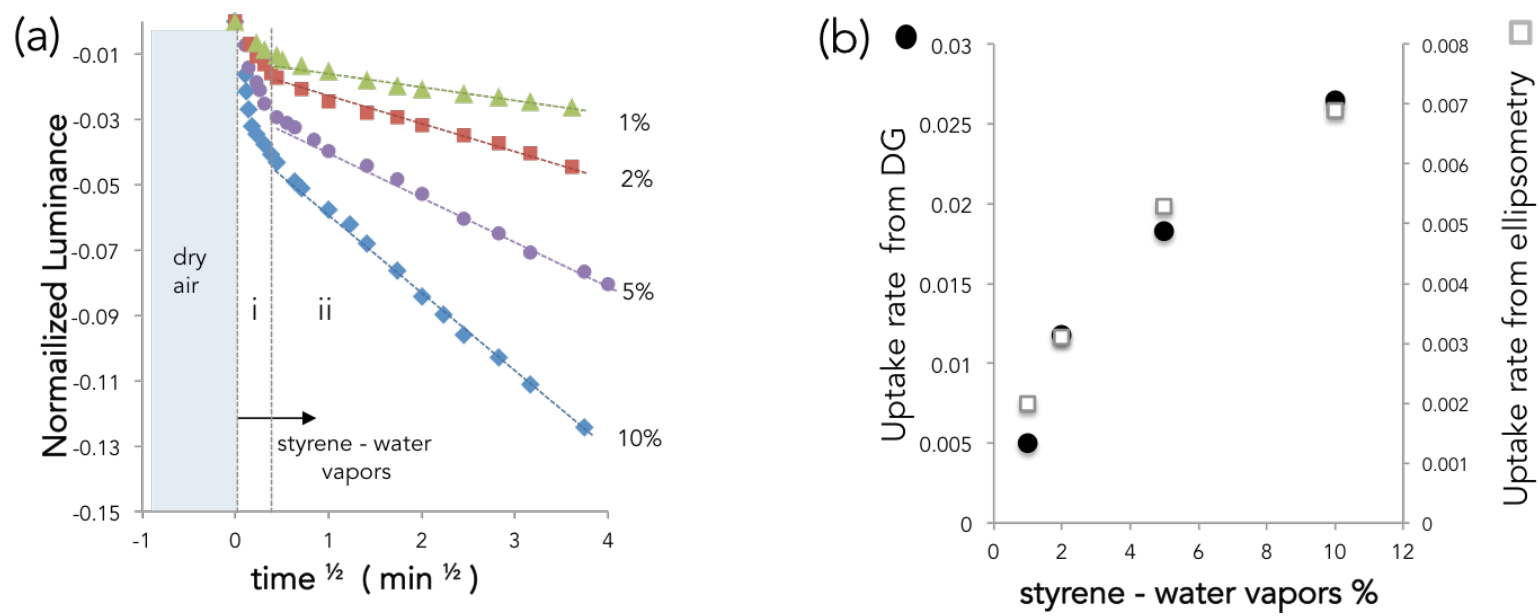

Figure 7 (a) Plot of the luminance of the $\mathrm{ZIF}-8 / \mathrm{TiO}_{2}$ diffraction grating with respect to the square root of time for styrene/water adsorptions at different vapors concentration. (b) Plot of the uptake rates versus vapor concentration obtained by diffraction grating (DG) and ellipsometry.

In order to verify if this approach could be generalized to other organic molecules, the experiment shown in Figure 6 (b) was performed with other organic solvents (Fig. S10). In particular, a ZIF-8 film was exposed to styrene, toluene, cyclohexane and xylene vapors at the same concentration of $5 \% \mathrm{P} / \mathrm{P}_{0}$. As shown in Figure $\mathrm{S} 10$ (left), for all the systems, the two regimes could be identified. The amplitude of the refractive index and the variation of the uptake rates determined by fitting the slope of regimes (i) and (ii) for each solvent are reported in the graph in Fig. S10 (right). A significant variation in uptake rates in both regimes is observed for the four solvents. The adsorption rate of the four volatile hydrocarbons are found in the following order for regime ii: cyclohexane $>$ xylene $>$ styrene $>$ toluene. This difference can be attributed to a certain number of factors including a molecular sieving effect and the different chemical affinity between the adsorbed solvent and the ZIF-8 particle surface (regime i) and the inner microporosity (regime ii). This solvent-dependent 
behavior suggests that a strategy based on the determination of the adsorption rates can be envisioned to evaluate the contribution of each component in the case of mixed solvents. This could be achieved by approaches of combinatorial sensing by the integration of different sensing materials in a single platform as reported in the literature. ${ }^{[8 \mathrm{c}]}$

\section{Conclusions}

In this work we demonstrated an versatile approach to fabricate MOF- based 2D photonic structures by soft NanoImprinting Lithography. ZIF-8 homo and $\mathrm{ZIF}-8 / \mathrm{TiO}_{2}$ hetero structures, exhibiting several features with dimensions as small as $200 \mathrm{~nm}$, were replicated on different substrates over relatively large surfaces (up to $1 \mathrm{~cm}^{2}$ ). The resulting systems exhibited iridescence characteristic of 2D diffraction gratings. A new sensing approach based on the variation of the optical properties of MOF patterns, induced by the selective adsorption of organic solvent vapors, was studied. This method relies on the evaluation of the adsorptioninduced-color-luminance change, induced by the MOF refractive index variation, which is simply detected by a CCD camera, as those integrated in simple smart-phones. Detection performances of the sensing platforms were demonstrated using isopropanol as a model adsorbate and a biphasic styrene/water mixture as a relevant environmental contaminant system. Vapors sensing was successfully demonstrated by using ZIF-8 as sensible matrix integrated into 2D diffraction gratings. Interestingly detection of styrene can be based on the dynamic variation of optical properties of the films and not on their absolute values. In this simple proof-of-concept, we didn't test vapors concentrations below $1 \%$ of styrene; in addition some improvements can be envisioned to improve the transduction method. Even in the case of the less concentrated vapors mixture $(1 \%)$, the content of styrene in the atmosphere was calculated being equal to $57 \mathrm{ppm}$, a value that is below the Permissible

Exposure Limit in the US for employees working in chemical industry; ${ }^{[35]}$ other styrene detection protocols mainly based on gas chromatography ${ }^{[35]}$ ensures better sensitivity but requires the utilization of bulky, expensive instrumentations. Importantly, this approach based of MOF diffraction gratings allows an easy optical detection by using a simple and accessible tool such as the camera of a smart phone that can be potentially interfaced with image analysis applications for real-time air-quality monitoring.

\section{Experimental Section}




\section{Master and PDMS-based stamp fabrication}

The silicon master mold were fabricated by electron beam lithography at $100 \mathrm{KeV}$ (Vistec 5000+) and reactive ion etching. An A7 PMMA positive-tone resist (495PMMA A Resists, solids: 7\% in Anisole) was spin-coated on a 2-inch $\mathrm{Si}(100)$ wafer and baked for $45 \mathrm{~min}$ at 160 ${ }^{\circ} \mathrm{C}$. The sample was exposed at $10 \mathrm{nA}$ with a dose of $1300 \mu \mathrm{C} / \mathrm{cm} 2$, developed for $45 \mathrm{sec}$ in a solution of Methyl-isobutyl-ketone (MIBK) and Isopropanol (mixture 1:3), rinsed in Isopropanol and gently dried with pure nitrogen gas. The pattern was transferred by reactive ion etching using a SF6/CHF3 gas mixture.

The anti-sticking treatment was performed using $1 \mathrm{H}, 1 \mathrm{H}, 2 \mathrm{H}, 2 \mathrm{H}-\mathrm{Perfluorooctyltriethoxysilane}$ (POTS) by chemical vapor deposition method. A sealed glass Petri dish containing the Silicon master and several drops of POTS was heated in an oven at about $120^{\circ} \mathrm{C}$ for $1 \mathrm{~h}$ to enable the reaction between the $\mathrm{OH}$ groups on the $\mathrm{Si}$ substrate surfaces and the POTS and then maintained at about $150{ }^{\circ} \mathrm{C}$ for $2 \mathrm{~h}$ to remove the unreacted POTS molecules. ${ }^{[36]}$

A bi-layer hard-PDMS/PDMS stamp was then replicated first by spin-coating a thin hardPDMS layer on the silicon master and secondly by casting a mixture of commercial available (RTV615 from GE) two components solution (1:10) on top. The bi-layer hard-PDMS/PDMS stamp was degassed and soft-baked at $60{ }^{\circ} \mathrm{C}$ for $48 \mathrm{~h}$. Finally, after being peeled off from the silicon master, the bi-layer stamp was treated with trichloromethylsilane TMCS. ${ }^{[37]}$

\section{ZIF-8 homo- and $\mathrm{ZIF}-8 / \mathrm{TiO}_{2}$ hetero structures fabrication}

The synthesis of the ZIF-8 nanoparticles followed the conditions reported by Cravillon et al. ${ }^{[25]}$ A solution of $\mathrm{Zn}\left(\mathrm{NO}_{3}\right)_{2} \cdot 6 \mathrm{H}_{2} \mathrm{O}(2.933 \mathrm{~g}, 9.87 \mathrm{mmol}$; $98 \%$ Sigma-Aldrich) in $200 \mathrm{~mL}$ of methanol (100\% VWR Chemicals) is poured in a solution of 2-methylimidazole $(6.489 \mathrm{~g}$, $79.04 \mathrm{mmol}$; 99\% Sigma-Aldrich) in $200 \mathrm{~mL}$ of methanol under stirring at room temperature. After $1 \mathrm{~h}$, the nanocrystals are separated from the milky solution by centrifugation at 20000 rpm for $15 \mathrm{~min}$. Nanoparticles were redispersed in absolute ethanol and centrifuged 3 times to remove the unreacted species.

For the patterning of ZIF-8 nanostructures, a $10 \mu \mathrm{L}$-droplet of colloidal solution was first applied on the substrate. The solution droplet was immediately covered by the patterned PDMS stamp without applying any external pressure. The liquid layer was homogeneously spread beneath the PDMS stamp while the stamp's nanocavities were naturally filled by capillarity. In this configuration, the evaporation of the solvent takes place through the permeable PDMS stamp. Replication of the features and homogeneous packing of the 
colloidal network were obtained after complete evaporation and de-molding. Finally, the system was consolidated at $130^{\circ} \mathrm{C}$ for 10 minutes.

For the fabrication of $\mathrm{ZIF}-8 / \mathrm{TiO}_{2}$ heterostructures, titania thin films were first prepared from a solution composed of $\mathrm{TiCl}_{4} / \mathrm{H}_{2} \mathrm{O} /$ Ethanol $\left(\mathrm{TiCl}_{4} 99 \%\right.$ Sigma-Aldrich) with a respective molar ratio of $1 / 6 / 50$. After complete dissolution and homogenization, a layer of solution was deposited on silicon wafers by spin-coating at $2500 \mathrm{rpm}$ for $10 \mathrm{~s}$ under a relative humidity of $40 \%$. In the same time, the PDMS stamp was pumped 10 min under vacuum and then readily applied on the as-deposited titania thin film. After $1 \mathrm{~min}$ at room temperature the thin film surrounded by the PDMS stamp was heated at $100^{\circ} \mathrm{C}$ for $5 \mathrm{~min}$ on a hot plate. The PDMS stamp was then removed and the titania thin film was finally calcinated at $450^{\circ} \mathrm{C}$ for $5 \mathrm{~min}$ on a hot plate. ZIF-8 coating was deposited on nanoimprinted $\mathrm{TiO}_{2}$ gratings by dip-coating with a withdrawal speed of $8 \mathrm{~mm} / \mathrm{s}$.

\section{Characterization}

Ellipsometry measurements were performed on a UV-visible (from 240 to $1000 \mathrm{~nm}$ ) variable angle spectroscopic ellipsometer (VASE - 2000U Woollam), and the data analyses were performed with the Wvase32 software using Cauchy models. Environmental ellipsometry porosimetry involves a dynamic in situ monitoring of the refractive index and the thickness of the film upon variation of the relative vapor pressures between $0 \%$ and $100 \%$ inside the analysis chamber. Microscope images showing the film structure were collected using a field-emission gun scanning electron microscopy (FEG-SEM - Hitachi SU7000 instrument). Grazing incidence wide-angle X-ray scattering (GI-WAXS) was recorded using a Rigaku SMAX 3000 with simultaneous small angle X-ray scattering (SAXS) capabilities, on an image plate.

\section{Detection experiments}

The photocamera-assisted detection experiment is schematically illustrated in Figure 4 and it was performed in a closed black box in order to avoid interferences with ambient light. Specific windows and connections were built for irradiation and detection and to allow vapors injection into the closed box. The diffracting grating samples were irradiated by a white LED (ZEISS CL 6000 LED) perpendicularly to the substrate. For each experiment, the intensity of the incident light was tuned between 0,1 and $3 \mathrm{~W} / \mathrm{cm}^{2}$ in order to achieve reasonable diffracted light avoiding saturation in the diffracted images. A commercially available smartphone I-Phone 3GS (photo camera resolution $3 \mathrm{MegaPixel)}$ ) was used for the i-PrOH detection while a steady photo-camera Manta G125CIRC (resolution 1.3 MegaPixels) was used for the 
longer styrene adsorption detection. The photo camera was place at an angle of $28^{\circ}$ as respect of the incident light and at distance of $30 \mathrm{~cm}$ from the sample.

\section{Supporting Information}

Supporting Information is available from the Wiley Online Library or from the author.

\section{Acknowledgements}

We thank David Montero for his support for electronic microscopy (Institut des Matériaux de Paris Centre FR2482). We acknowledge the financial support from CNano Region Ile-deFrance and the Labex Matisse.

\section{References}

[1] N. Stock, S. Biswas, Chemical Reviews 2012, 112, 933-969.

[2] J. R. Li, R. J. Kuppler, H. C. Zhou, Chem. Soc. Rev. 2009, 38, 1477-1504.

[3] a) X.-Z. Song, S.-Y. Song, S.-N. Zhao, Z.-M. Hao, M. Zhu, X. Meng, L.-L. Wu, H.-J. Zhang, Advanced Functional Materials 2014, 24, 4034-4041; b) S.-N. Zhao, L.-J. Li, X.-Z. Song, M. Zhu, Z.-M. Hao, X. Meng, L.-L. Wu, J. Feng, S.-Y. Song, C. Wang, H.-J. Zhang, Advanced Functional Materials 2015, 25, 1463-1469.

[4] M. Faustini, J. Kim, G.-Y. Jeong, J. Y. Kim, H. R. Moon, W.-S. Ahn, D.-P. Kim, Journal of the American Chemical Society 2013, 135, 14619-14626.

[5] a) P. Falcaro, R. Ricco, C. M. Doherty, K. Liang, A. J. Hill, M. J. Styles, Chem. Soc. Rev. 2014, 43, 5513-5560; b) S. Furukawa, J. Reboul, S. Diring, K. Sumida, S. Kitagawa, Chem. Soc. Rev. 2014, 43, 5700-5734.

[6] a) A. Demessence, C. Boissiere, D. Grosso, P. Horcajada, C. Serre, G. Ferey, G. Soler-Illia, C. Sanchez, Journal of Materials Chemistry 2010, 20, 7676-7681; b) A. Demessence, P. Horcajada, C. Serre, C. Boissiere, D. Grosso, C. Sanchez, G. Ferey, Chem. Commun. 2009, 7149-7151.

[7] G. Lu, J. T. Hupp, Journal of the American Chemical Society 2010, 132, 7832-7833.

[8] a) F. M. Hinterholzinger, A. Ranft, J. M. Feckl, B. Ruhle, T. Bein, B. V. Lotsch, Journal of Materials Chemistry 2012, 22, 10356-10362; b) Z. Hu, C.-a. Tao, F. Wang, X. Zou, J. Wang, Journal of Materials Chemistry C 2015, 3, 211-216; c) A. Ranft, F. Niekiel, I. Pavlichenko, N. Stock, B. V. Lotsch, Chemistry of Materials 2015, 27, 1961-1970.

[9] G. Lu, O. K. Farha, L. E. Kreno, P. M. Schoenecker, K. S. Walton, R. P. Van Duyne, J. T. Hupp, Adv. Mater. 2011, 23, 4449-+.

[10] O. Shekhah, J. Liu, R. A. Fischer, C. Woll, Chem. Soc. Rev. 2011, 40, 1081-1106.

[11] A. Cattoni, P. Ghenuche, A.-M. Haghiri-Gosnet, D. Decanini, J. Chen, J.-L. Pelouard, S. p. Collin, Nano Letters 2011, 11, 3557-3563.

[12] D. Chanda, K. Shigeta, S. Gupta, T. Cain, A. Carlson, A. Mihi, A. J. Baca, G. R. Bogart, P. Braun, J. A. Rogers, Nat Nano 2011, 6, 402-407.

[13] P. Falcaro, D. Buso, A. J. Hill, C. M. Doherty, Adv. Mater. 2012, 24, 3153-3168.

[14] a) Y. You, H. Yang, J. W. Chung, J. H. Kim, Y. Jung, S. Y. Park, Angewandte Chemie International Edition 2010, 49, 3757-3761; b) R. Ameloot, E. Gobechiya, H. Uji-i, J. 
A. Martens, J. Hofkens, L. Alaerts, B. F. Sels, D. E. De Vos, Adv. Mater. 2010, 22, 2685.

[15] D. Witters, N. Vergauwe, R. Ameloot, S. Vermeir, D. De Vos, R. Puers, B. Sels, J. Lammertyn, Adv. Mater. 2012, 24, 1316-1320.

[16] R. Ameloot, L. Stappers, J. Fransaer, L. Alaerts, B. F. Sels, D. E. De Vos, Chemistry of Materials 2009, 21, 2580-2582.

[17] S. Hermes, F. Schroder, R. Chelmowski, C. Woll, R. A. Fischer, Journal of the American Chemical Society 2005, 127, 13744-13745.

[18] P. Falcaro, A. J. Hill, K. M. Nairn, J. Jasieniak, J. I. Mardel, T. J. Bastow, S. C. Mayo, M. Gimona, D. Gomez, H. J. Whitfield, R. Ricco, A. Patelli, B. Marmiroli, H. Amenitsch, T. Colson, L. Villanova, D. Buso, Nat. Commun. 2011, 2, 8.

[19] a) C. Carbonell, I. Imaz, D. Maspoch, Journal of the American Chemical Society 2011, 133, 2144-2147; b) E. Bellido, S. Cardona-Serra, E. Coronado, D. Ruiz-Molina, Chem. Commun. 2011, 47, 5175-5177.

[20] C. Dimitrakakis, B. Marmiroli, H. Amenitsch, L. Malfatti, P. Innocenzi, G. Grenci, L. Vaccari, A. J. Hill, B. P. Ladewig, M. R. Hill, P. Falcaro, Chem. Commun. 2012, 48, 7483-7485.

[21] a) G. Lu, O. K. Farha, W. N. Zhang, F. W. Huo, J. T. Hupp, Adv. Mater. 2012, 24 , 3970-3974; bB. K. Keitz, C. J. Yu, J. R. Long, R. Ameloot, Angew. Chem.-Int. Edit. 2014, 53, 5561-5565.

[22] C. M. Doherty, G. Grenci, R. Ricco, J. I. Mardel, J. Reboul, S. Furukawa, S. Kitagawa, A. J. Hill, P. Falcaro, Adv. Mater. 2013, 25, 4701-4705.

[23] B. Chen, Z. Yang, Y. Zhu, Y. Xia, Journal of Materials Chemistry A 2014, 2, 16811 16831.

[24] K. S. Park, Z. Ni, A. P. Côté, J. Y. Choi, R. Huang, F. J. Uribe-Romo, H. K. Chae, M. O'Keeffe, O. M. Yaghi, Proceedings of the National Academy of Sciences 2006, 103, 10186-10191.

[25] J. Cravillon, S. Münzer, S.-J. Lohmeier, A. Feldhoff, K. Huber, M. Wiebcke, Chemistry of Materials 2009, 21, 1410-1412.

[26] C. Luo, X. Ni, L. Liu, S.-i. M. Nomura, Y. Chen, Biotechnology and Bioengineering 2010, 105, 854-859.

[27] C. S. Chern, Progress in Polymer Science 2006, 31, 443-486.

[28] M. Zhu, X.-Z. Song, S.-Y. Song, S.-N. Zhao, X. Meng, L.-L. Wu, C. Wang, H.-J. Zhang, Advanced Science 2015, 2, n/a-n/a.

[29] J. F. Feller, H. Guezenoc, H. Bellegou, Y. Grohens, Macromol. Symp. 2005, 222, 273 280.

[30] Y. Wang, X. Du, Y. Long, X. Tang, Z. Chen, Y. Jiang, Sensors and Actuators B: Chemical 2015, 206, 252-257.

[31] E. T. Zellers, N. Hassold, R. M. White, S. M. Rappaport, Analytical Chemistry 1990, $62,1227-1232$.

[32] M. P. Rowe, W. H. Steinecker, E. T. Zellers, Analytical Chemistry 2007, 79, $1164-$ 1172 .

[33] Y. Li, J. N. Ding, N. Y. Yuan, in Micro-Nano Technology Xiv, Pts 1-4, Vol. 562-565 (Ed.: F. Tang), Trans Tech Publications Ltd, Stafa-Zurich, 2013, pp. 446-451.

[34] P. Pandey, J. K. Srivastava, V. N. Mishra, R. Dwivedi, J. Nat. Gas Chem. 2011, 20, 123-127.

[35] $100 \mathrm{ppm}$ according to the Occupational Safety \& Health Administration : https://www.osha.gov/pls/oshaweb/owadisp.show_document?p_table=STANDARDS $\& p \_i d=9993$ 
[36] L. Wang, J. Wei, Z. Su, Langmuir 2011, 27, 15299-15304.

[37] A. Cattoni, E. Cambril, D. Decanini, G. Faini, A. M. Haghiri-Gosnet, Microelectronic Engineering 2010, 87, 1015-1018. 